\title{
Effects of chitosan coating on physical properties and pharmacokinetic behavior of mitoxantrone liposomes
}

\author{
This article was published in the following Dove Press journal: \\ International Journal of Nanomedicine \\ 18 June 2010 \\ Number of times this article has been viewed
}

\section{Jie Zhuang' \\ Qineng Ping' \\ Yunmei Song ${ }^{2}$ \\ Jianping $\mathrm{Qi}^{1}$ \\ Zheng Cui ${ }^{3}$}

'School of Pharmacy, China Pharmaceutical University, Nanjing, China; ${ }^{2}$ School of Pharmacy and

Medical Sciences, University of South Australia, Adelaide, Australia; ${ }^{3}$ School of Pharmacy, Peking University, Beijing, China
Correspondence: Qineng Ping

China Pharmaceutical University,

24 Tong Jia Xiang, Nanjing 210009 ,

China

Tel +86258327 I092

Email pingqn@cpu.edu.cn

\begin{abstract}
The objective of this work was to evaluate the physical properties and in vivo circulation of chitosan (CH)-coated liposomes of mitoxantrone (MTO). Changes in particle size and zeta potential confirmed the existence of a coating layer on the surface of liposomes. The in vitro release of adsorbed $\mathrm{CH}$ from the liposomes was significantly slower than $\mathrm{CH}$ solution, indicating the stable interaction between $\mathrm{CH}$ and liposomes. The physical stability of the $\mathrm{CH}$-coated liposomes was evaluated by measuring the change in particle size before and after freeze-drying and rehydration. The smallest change was observed when saturated adsorption of $\mathrm{CH}$ occurred $(0.3 \%)$. The sustained release in vitro of MTO from $\mathrm{CH}$-coated liposomes confirmed the increased stability of liposomes. Systemic circulation of $\mathrm{CH}$-coated MTO liposomes was examined. The $0.3 \% \mathrm{CH}$-coated liposomes showed the longest circulation time. It could be concluded that the prolonged retention time of the liposomes was closely related with $\mathrm{CH}$ coating and its stability effect.
\end{abstract}

Keywords: liposomes, chitosan coating, stability, in vitro release, prolonged retention time

\section{Introduction}

Liposomes are promising drug carriers because of their increased therapeutic efficacy and decreased toxicity compared with the drug prototype. Liposomes for anti-tumor drugs can passively accumulate in tumor tissues as a result of the enhanced permeability of tumor vasculature, thereby limiting drug distribution to normal tissues, which also helps decrease drug toxicity. ${ }^{1,2}$ However, the stability problem of liposomes in vitro and in vivo limits their application and development as liposomes tend to degrade or aggregate and fuse, which leads to the leakage of entrapped drug during storage ${ }^{3}$ and rapid clearance from the circulation system after intravenous injection. ${ }^{4}$ Many studies have found that, among all the factors affecting the stability of liposomes, surface characteristics of the carriers, such as hydrophobicity, ${ }^{4-6}$ charge, ${ }^{7,8}$ and fluidity, ${ }^{9}$ are of great importance. The mechanisms explaining their effect are associated with the inter-molecular interaction and the interaction with various plasma components. ${ }^{10}$ Therefore, the simple surface modification of the carriers using polymers with specific properties may be an easy approach to modulating their in vitro and in vivo stability.

Polymer coating is a promising way of modifying the surface characteristics of liposomes, which simply mixes a liposome suspension with a polymer solution without chemically linking the polymers to the lipid molecules. ${ }^{11}$ By coating the liposomes with polymers, the stability of liposomes can be improved during storage due to the long-range mutual repulsion between adjacent bilayers. It has been reported that a variety of polymers have been incorporated into the modification of liposomes, 
including natural components such as polysaccharides, or synthetic polymers such as polyethylene, polyvinyl alcohol, or polyacrylamide, ${ }^{11-13}$ among which $\mathrm{CH}$, a positively charged polysaccharide, is one with a promising future in the pharmaceutical and medical fields. $\mathrm{CH}((1 \rightarrow 4)$ 2 -amino-2deoxy- $\beta$-D-glucan), a deacetylated derivative of chitin, is an abundant and natural carbohydrate polymer. $\mathrm{CH}$ is regarded as nontoxic, biocompatible, biodegradable, ${ }^{14}$ and is widely used as a useful excipient in various drug delivery systems. To date, $\mathrm{CH}$ has been mainly applied to coat liposomes as a mucoadhesive polymer, and as a permeation enhancer for non-invasive drug delivery due to its ability to open epithelial tight junctions to allow for an increase in paracellular transport of macromolecular drugs. Another major application for $\mathrm{CH}$ is to form complex with vaccines or plasmid DNA in non-viral gene therapy. ${ }^{15}$ Studies on $\mathrm{CH}$-coated liposomes as anti-tumor drug carriers for injection use are rare. In addition, most papers focused only on the in vitro properties of $\mathrm{CH}$-coated liposomes, with few dealing with the systemic evaluation of them. In this paper, we tried to give an overall investigation on the in vitro and in vivo stability of $\mathrm{CH}$-coated liposomes.

In this study, MTO, a synthetic anthraquinone derivative, was chosen as a model drug. MTO has been extensively used for the treatment of various cancers; however, the dose-related cardiotoxicity and myelosuppression of anthraquinone drugs limit their clinical use to a large extent. In recent years, preparations of MTO liposomes have been developed in an effort to decrease its adverse effects. ${ }^{16}$ Clinically, MTO is of special effectiveness in the treatment of acute myelogenous leukemia. Therefore, the $\mathrm{CH}$-coated MTO-containing liposomes are expected to enhance storage stability and prolong systemic circulation.

The objectives of the present work were: first, to obtain and characterize liposomes coated with $\mathrm{CH}$ by measuring the particle size and the zeta potential and second, to give a thorough investigation of the effect of polymer coating on in vitro and in vivo behavior of the obtained $\mathrm{CH}$-coated liposomes by evaluating the physical stability through freeze-drying followed by rehydration (FD-RH) and the systemic circulation of the liposomes. More specifically, for the first time, we evaluated the in vitro desorption of $\mathrm{CH}$ from the liposomes using $\mathrm{CH}$ solution as control, further clarifying the lasting protective effect of $\mathrm{CH}$ for liposomes after entering systemic circulation in vivo in rat.

\section{Methods and materials}

Soybean phospholipid was a kind gift from Evonik Degussa China Co., Ltd (Shanghai, China) (purity $>90 \%$ ). Cholesterol was purchased from Huixing Biochemical Reagent Co., Ltd (Shanghai, China). CH (degree of deacetylation: 92\%, viscosity average molecular weight: 70,000 Da) was provided by Nantong Shuanglin Biochemical Co., Ltd. (Jiangsu, China). Mitoxantrone dihydrochloride (99.1\%) was obtained from Sichuan Shenghe Pharmaceutical Co., Ltd. Methanol was of HPLC grade. Other reagents were of analytical grade. Water was deionized before use.

\section{Preparation of liposomes and $\mathrm{CH}$-coated liposomes}

Liposomes containing MTO were prepared by an ammonium sulfate gradient loading procedure. ${ }^{17}$ Unloaded liposomes composed of lipids and cholesterol (5:1 in molar ratio) were prepared by thin film hydration followed by sonication. Briefly, $200 \mathrm{mg}$ of PC and $20 \mathrm{mg}$ of cholesterol were dissolved in a small amount of a mixture solution of methanol and chloroform, and the solution was rotary evaporated at $37^{\circ} \mathrm{C}$ under reduced pressure to obtain a thin lipid film. The thin lipid film was dried in vacuum overnight to ensure complete removal of the solvent and hydration was carried out with $10 \mathrm{~mL}$ of aqueous ammonium sulfate $(0.3 \mathrm{M})$ using a vortex mixer. The resultant multilamellar liposomal suspension was sonicated at $4^{\circ} \mathrm{C}$ using a micro-tip probe sonicator, JY 92-II ultrasonic processor (Syklon, Zhejiang, China) to obtain small unilamellar liposomes. After sonication, the unloaded liposomes were dialyzed against normal saline to remove the extraliposomal ammonium sulfate. The drug-loading liposomes were prepared by incubating the solution of MTO $(4 \mathrm{mg} / \mathrm{mL})$ with the suspension of the unloaded liposomes at $37^{\circ} \mathrm{C}$ for $20 \mathrm{~min}$.

For preparation of $\mathrm{CH}$-coated liposomes, an appropriate amount of $\mathrm{CH}$ was dissolved in $1 \% \mathrm{v} / \mathrm{v}$ of acetic acid solution and diluted with sodium acetate buffer solution $(\mathrm{pH}$ 6.0) to various concentrations $(0.1 \%, 0.3 \%, 0.6 \%$ and $1.2 \%, \mathrm{w} / \mathrm{v})$. Then, liposome suspension was added dropwise to $\mathrm{CH}$ solution of equal volume. The mixed solution was incubated at $10^{\circ} \mathrm{C}$ for 1 hour with continuous stirring. The final concentrations of the lipids and $\mathrm{CH}$ were half of the original solutions, and the final MTO concentration in the $\mathrm{CH}$-coated liposomes was $0.5 \mathrm{mg} / \mathrm{mL}$.

\section{Characterization of $\mathrm{CH}$-coated liposomes}

Particle size and zeta potential of $\mathrm{CH}$-coated liposomes were measured by a Zetasizer 3000 HS (Malvern Instruments, Malvern, UK). Samples for transmission electron microscopy (TEM) were prepared at room temperature by 
conventional negative staining using $0.3 \%$ phosphotungstic acid buffer ( $\mathrm{pH}$ 6.0). Samples were viewed on a transmission electron microscope H 7650 (Hitachi High-Technologies, Tokyo, Japan).

\section{Content and entrapment efficiency of drug}

Concentration of MTO was determined by HPLC. The HPLC system (Shimadzu Corporation, Kyoto, Japan) consisted of a LC-10AT pump and a SPD-10A UV detector set at $658 \mathrm{~nm}$. The analyte was determined at room temperature on a $150 \mathrm{~mm} \times 4.6 \mathrm{~mm}$ Diamonsil ${ }^{\circledR} \mathrm{C} 18$ column (Dikma Technologies, Beijing, China). The mobile phase consisted of methanol - ammonium acetate buffer solution (20 mM) $(48: 52 \mathrm{v} / \mathrm{v})$. The ammonium acetate buffer solution was adjusted to $\mathrm{pH} 2.0$ with sulfuric acid before mixed with methanol. The mobile phase was pumped through the system at a rate of $1 \mathrm{~mL} / \mathrm{min}$.

The total drug content of suspensions was determined by dissolving liposomes in appropriate amount of methanol containing $1 \%(\mathrm{v} / \mathrm{v}) 1 \mathrm{M} \mathrm{HCl}$ (acidified methanol) and measured by HPLC.

The entrapment efficiency of the liposomes was determined using a gel filtration method. Free drug was separated from encapsulated MTO over a Sephadex G-25 $(1.4 \mathrm{~cm} \times 35 \mathrm{~cm})$ column. MTO-loaded liposomal suspension $(0.5 \mathrm{~mL})$ was added to the column. The encapsulated drug was eluted with $5 \%$ glucose solution and then the free drug was eluted with normal saline. One milliliter of the liposome elute was diluted with acidified methanol for HPLC and the eluted free drug was injected into the HPLC system directly. The total drug content of the suspensions was also determined. The encapsulation efficiency (EE) was calculated using Eq. (1):

$$
\mathrm{EE}(\%)=\mathrm{AUCE} / \mathrm{AUCT} \times 100
$$

where AUCE and AUCT are the area under the elution profile curve of the encapsulated and total drug in the feed, respectively. ${ }^{18}$

\section{Coating efficiency of $\mathrm{CH}$}

Aliquots of $\mathrm{CH}$-coated liposome suspensions $(0.4 \mathrm{~mL})$ were centrifuged at 12,000 rpm for $5 \mathrm{~min}$ in an ultimate filtration tube (molecular weight cut 10,000). The non-adhered $\mathrm{CH}$ at the bottom of the tube was determined by a colorimetric method described by Gao et al with a slight modification. ${ }^{19}$ A solution of Alizarin Red S was prepared in deionized water, at a concentration of $1.5 \mathrm{mg} / \mathrm{mL}$. To prepare the standard curve, a stock solution of $\mathrm{CH}$ was prepared at a concentration of $1 \mathrm{mg} / \mathrm{mL}$. Different volumes of the stock solution were transferred into volumetric flasks. Aliquots of the dye solution $(0.8 \mathrm{~mL})$ were added to each flask, after which the volume in each flask was filled to $10 \mathrm{~mL}$ with sodium acetate buffer solution at a $\mathrm{pH}$ of 5.0. The UV adsorption was detected at $580 \mathrm{~nm}$ with an $8453 \mathrm{UV}$-Visible Spectrophotometer (Agilent Technologies, Santa Clara, CA, USA) within 2 hours.

\section{In vitro desorption of $\mathrm{CH}$ from liposomes}

The aim of the study was to investigate the desorption of $\mathrm{CH}$ from the surface of the liposomes. To protect the liposomes from deteriorating by the plasma components, $\mathrm{CH}$ should not be removed immediately on dilution after entering the systemic circulation. In this experiment, $0.3 \% \mathrm{CH}$ solution was first diluted with sodium acetate buffer solution at $\mathrm{pH} 6.0$ to half of the original concentration and used as control and CH (0.3\%)-coated liposomes without MTO were first prepared according to the method described in "Preparation of liposomes and CH-coated liposomes." Thereafter, $4 \mathrm{~mL}$ of the $\mathrm{CH}$-coated liposomes or $4 \mathrm{~mL}$ of $\mathrm{CH}$ solution was respectively placed in a cellulose membrane tubing (molecular weight cut-off was 10,000, membrane pore size $10 \mathrm{~nm}$ ). Then the tubing was tightened and soaked in $40 \mathrm{~mL}$ of phosphate buffer solution ( $\mathrm{pH}$ 7.4) as dissolution medium. The experiments were carried out at $37^{\circ} \mathrm{C}$ for 8 hours in 3 replicates. At predetermined intervals, $0.5 \mathrm{~mL}$ of the dissolution medium was withdrawn and replaced with the same amount of prewarmed dissolution medium. The samples of the replicates were pipetted directly into disposable cuvettes and analyzed for $\mathrm{CH}$ content as described in "Coating efficiency of $\mathrm{CH}$."

Statistical analysis was performed using Student's t-test with $P<0.05$ as the minimal level of significance on the raw data of the release rate of $\mathrm{CH}$ solution and adsorbed $\mathrm{CH}$ from liposomes at each time point.

\section{Physical stability studies}

The liposomal suspension was subjected to FD-RH with water to assess the physical stability of the liposomes. One $\mathrm{mL}$ of liposomal suspension was preparatively frozen at $-70^{\circ} \mathrm{C}$ and applied to the LGJ-10 Freeze-Dry Machine, (Songyuan Huaxing Technology Development Co., Ltd., Beijing, China) for 24 hours. The freeze-dried cake was rehydrated with $1 \mathrm{~mL}$ of distilled water containing equal amount of $1 \%$ acetic acid (for uncoated liposomes, with water) by vortex-mixing till the cake was optically clear. The particle size of the dispersed 
liposomes was measured with a Zetasizer 3000 HS (Malvern Instruments, Malvern, UK).

\section{In vitro drug release}

In vitro release of MTO from liposomal formulations was analyzed by membrane dialysis. Pre-experiments were carried out on the in vitro release of MTO from liposomes with or without $\mathrm{CH}$ coating in phosphate buffer solution ( $\mathrm{pH} 7.4$ ), and less than $5 \%$ of MTO was released up to 48 hours from each preparation (data not shown). In this study, equal amount of fetal cattle serum (FCS) was added to the liposome to simulate the in vivo environment. Briefly, $4 \mathrm{~mL}$ of CH-coated liposomes mixed with $4 \mathrm{~mL}$ of FCS was placed in the cellulose membrane tubing (molecular weight cut-off was 8,000-10,000). For uncoated liposomes, sodium acetate buffer solution ( $\mathrm{pH}$ 6.0) was added to the liposomes to adjust the concentration. Meanwhile, MTO aqueous solution was treated in the same way and used as control. Then the tubing was tightened and soaked in $200 \mathrm{~mL}$ of PBS 7.4 as dissolution medium. The experiments were carried out at $37^{\circ} \mathrm{C}$ for 48 hours in 3 replicates. At predetermined intervals, $5 \mathrm{~mL}$ of the dissolution medium was withdrawn and replaced with the same amount of pre-warmed dissolution medium. The samples of the replicates were pipetted directly into disposal cuvettes and analyzed by an $8453 \mathrm{UV}$-Visible Spectrophotometer (Agilent Technologies, Santa Clara, CA, USA) at $658 \mathrm{~nm}$.

\section{In vivo studies}

\section{Animals}

Wistar rats (200-240 g, male), obtained from the animal center of China Pharmaceutical University (Nanjing, China), were maintained under uniform experimental conditions (temperature $25 \pm 2{ }^{\circ} \mathrm{C}$; humidity $60 \pm 5 \%, 12$ hours dark/ light cycle) for 1 week prior to experiments. They were fed with standard laboratory chow. All rats were fasted overnight before the experiment while water was provided ad libitum. All animal treatments followed the recommendations of the Regulations for the Administration of Affairs Concerning Experimental Animals.

\section{Drug administration and blood sampling}

Four groups of 6 rats each were treated with the normal saline solution of MTO, uncoated liposomes and $\mathrm{CH}$-coated liposomes $(0.3 \%$ and $0.6 \%, \mathrm{w} / \mathrm{v})$ at a single MTO dose of $13 \mathrm{mg} / \mathrm{m}^{2}$ via femoral vein injection, respectively. At each time point $(5,15$, and 30 minutes and 1, 2, 4, 6, and 8 hours), blood samples $(0.3 \mathrm{~mL})$ were collected by oculi chorioidea vein into pre-labeled chilled micro-tubes containing heparin as an anticoagulant. Immediately after collection, each blood sample was gently inverted several times to ensure complete mixing with the anticoagulant. The blood samples were centrifuged for 10 minutes at $4000 \times \mathrm{g}$ to separate plasma, and the latter was transferred to cryotubes and stored at $-20^{\circ} \mathrm{C}$ until analysis.

\section{Sample preparation}

Plasma containing MTO was extracted by previously reported protein precipitation with slight modification. ${ }^{20}$ One hundred $\mu \mathrm{L}$ of plasma was mixed with $200 \mu \mathrm{L}$ of extraction solution (methanol containing $0.5 \mathrm{M}$ hydrochloric acid: acetonitrile (90:10, v:v)). After vigorous vortexing, the samples were centrifuged for 10 minutes at $12,000 \times \mathrm{g}$. The supernatants were removed and $20 \mu \mathrm{L}$ was injected into the HPLC column for analysis.

\section{Data analysis}

Plasma concentrations versus time data were analyzed by Kinetica 4.4 (Thermo Electron Corporation, Waltham, MA, USA). Parameters including AUC, MRT, and $t_{1 / 2}$ were determined.

A one-way ANOVA analysis was performed with $P<0.05$ as the minimal level of significance.

\section{Results and discussion Morphological shape}

The morphological appearance of $\mathrm{CH}$-coated liposomes and uncoated liposomes was visualized using TEM (Figure 1). It could be seen that both types of liposomes presented a spherical morphology, yet no significant morphological differences between liposomes with or without $\mathrm{CH}$ coating were observed. This is probably due to the strong adsorption between the polymer and the liposomal bilayer, resulting in a flat configuration and making it difficult to observe the polymer at the liposome surface. The same situation has been reported by others. ${ }^{21,22}$

\section{Particle size and zeta potential}

The liposomes were coated with $\mathrm{CH}$ at various concentrations in the solution. The particle size of the liposomes is shown in Figure 2. For measuring the particle size, liposomes were diluted by 20 -fold with water. The particle size of $\mathrm{CH}$-coated liposomes increased with the increased concentration of polymer solution, suggesting the formation of coating layer on the surface of the liposomes, and an increase in the thickness of the coating layer with the increase of the polymer 


\section{A)}

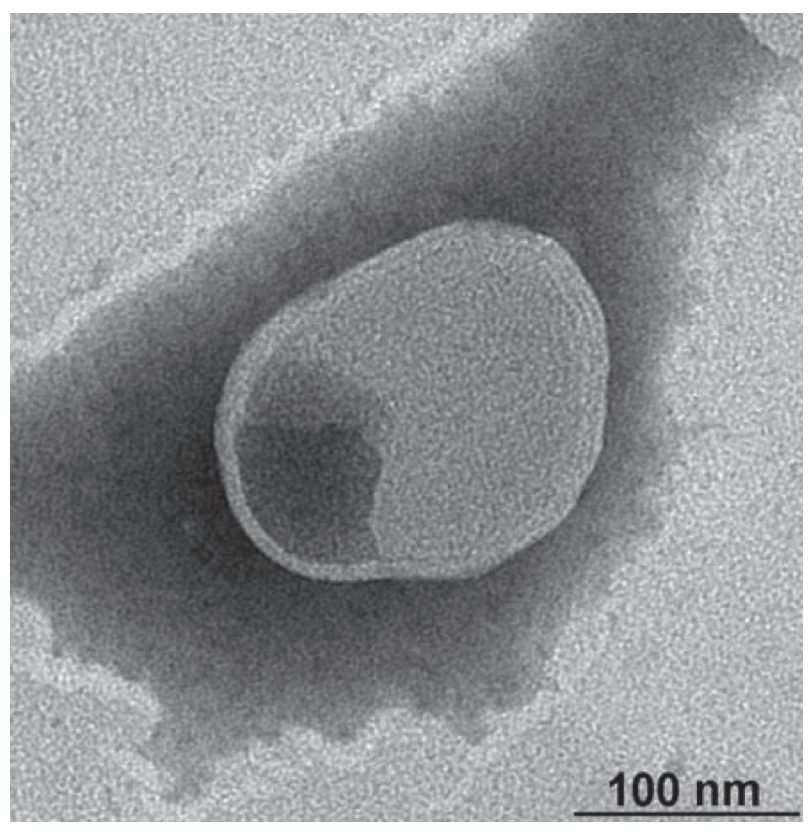

B)

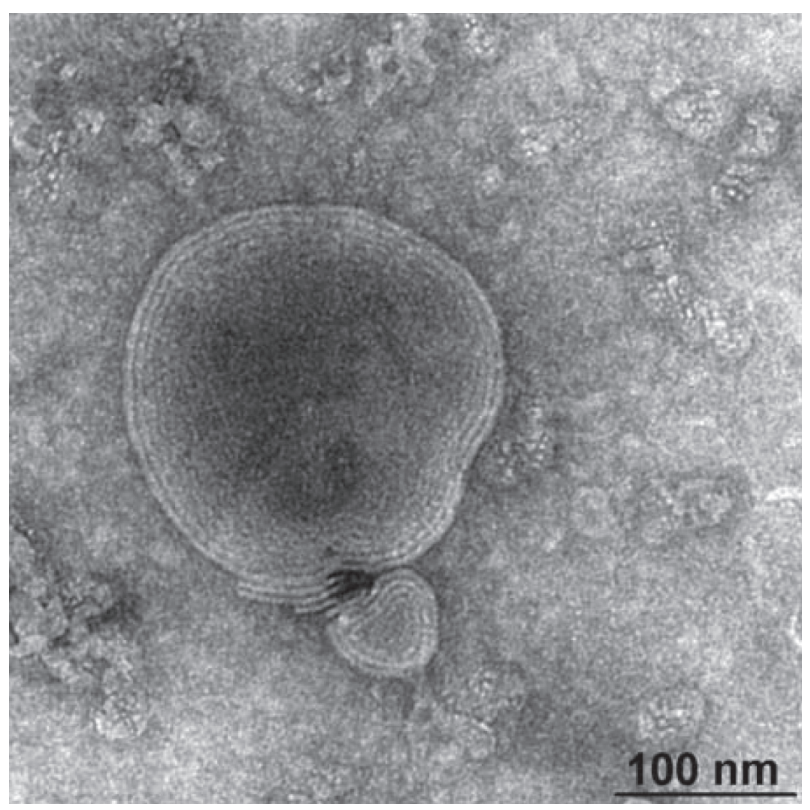

Figure I Transmission electron micrographs of A) uncoated liposomes and B) $\mathrm{CH}$-coated liposomes. Bar is $100 \mathrm{~nm}$.

concentration. However, the size did not change much before and after coating, only 1.34-fold as large as the original size even at the highest concentration of $\mathrm{CH}(1.2 \%, \mathrm{w} / \mathrm{v})$. The small change in particle size of liposomes is preferable for circulation, since larger particles tend to be removed from the blood by the cells of the mononuclear phagocyte system. ${ }^{23}$ The presence of strong intra-molecular electrostatic interactions is an explanation for the thin layer formed around the liposomes. ${ }^{24}$ After adsorption, the strong attraction between segments of the polyelectrolyte chains and oppositely charged groups on the surface forces them to come into close proximity, forming a thin layer around the surface..$^{25}$

The uncoated liposome had a marked negative charge $(-30.4 \mathrm{mv})$ which, after the incubation with $\mathrm{CH}$ at various concentrations $(0.02 \%, 0.04 \%, 0.2 \%, 0.3 \%, 0.6 \%$, and $1.2 \%$ $\mathrm{w} / \mathrm{v})$, was inverted to positive value. This inversion of the surface charge of the liposomes confirmed the presence of $\mathrm{CH}$ coating on the surface of the liposomes. Therefore, the main interaction between liposomes and $\mathrm{CH}$ was electrostatic attraction. The zeta potential reached a relatively constant value as the $\mathrm{CH}$ concentration increased to $0.3 \%$ (Figure 3 ), indicating the saturated adsorption of $\mathrm{CH}$ to liposomes.

\section{Entrapment efficiency of MTO}

The entrapment efficiency of MTO was $97.4 \%$ for uncoated liposomes. The addition of $\mathrm{CH}$ decreased the entrapment efficiency slightly (Figure 4), probably due to the interaction of $\mathrm{CH}$ with the apolar head groups on the surface of phospholipid bilayers. When the concentration of $\mathrm{CH}$ increased to $0.3 \%$, no more leakage of MTO was observed. Since saturated adsorption has achieved (see "Particle size and zeta potential") and the stable coating layer formed, the excess $\mathrm{CH}$ could only surround the outside of the liposomes and were not able to interfere with the bilayer. A similar phenomenon has been reported previously. ${ }^{26}$

\section{Coating efficiency of $\mathrm{CH}$}

The maximum adsorption of $\mathrm{CH}$ to the liposomes was achieved at a polymer concentration of $0.3 \%$ (see "Particle size and zeta potential"). The coating efficiency at maximum adsorption was estimated by measuring the concentration of free $\mathrm{CH}$ after centrifugation. The coating efficiency at maximum adsorption is $82 \%$, and when calculated on a weight basis, the weight ratio of $\mathrm{CH}$ to phospholipids is $1 / 8.13$ at a polymer concentration of $0.3 \%$.

\section{In vitro desorption of $\mathrm{CH}$ from liposomes}

Figure 5 depicts the $\mathrm{CH}$ desorption profile from the liposomes using $\mathrm{CH}$ solution as control. Both adsorbed $\mathrm{CH}$ and $\mathrm{CH}$ solution released $30 \%$ of their total amount within the first $5 \mathrm{~min}$. After that, the release rate of adsorbed $\mathrm{CH}$ from liposomes was significantly slower than that of $\mathrm{CH}$ solution $(P<0.05)$. Maximum release of $\mathrm{CH}$ was obtained both at $8 \mathrm{~h}$ with a release of $80 \%$ of adsorbed $\mathrm{CH}$ from liposomes in contrast to $100 \%$ of $\mathrm{CH}$ solution. $\mathrm{CH}$ combines with the phospholipid 


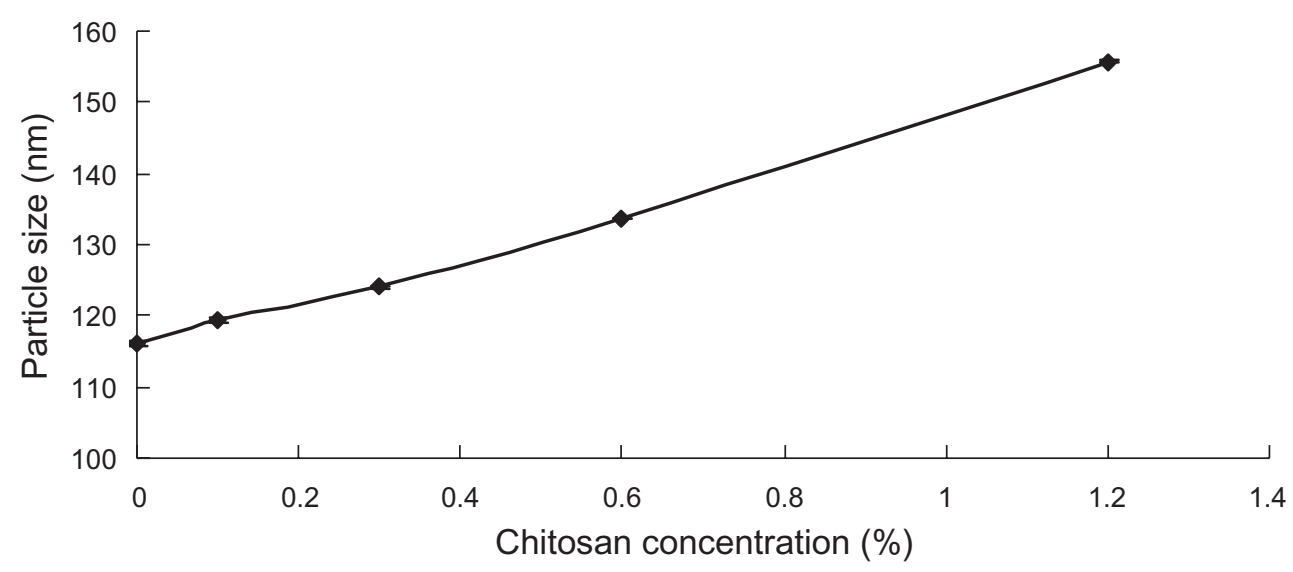

Figure 2 The influence of $\mathrm{CH}$ concentration on the size of $\mathrm{CH}$-coated liposomes $(n=3)$.

bilayers mainly through electrostatic interactions. In addition, CH's hydrophobic side chains can also exert hydrophobic interaction with the phospholipid bilayers. ${ }^{27}$ Due to the effect of the two kinds of interactions, the release of adsorbed $\mathrm{CH}$ was mostly retarded over the period. Therefore, it is reasonable that $\mathrm{CH}$ can still protect the liposomes after entering the systemic circulation, and won't be easily removed through dilution. The initial rapid release of adsorbed $\mathrm{CH}$ in the first 5 minutes was probably due to the free $\mathrm{CH}$ molecules existing in the preparation, as shown by $82 \%$ coating efficiency of $\mathrm{CH}(0.3 \%)$.

\section{Physical stability of $\mathrm{CH}$-coated liposomes}

The physical stability of the liposomes was evaluated by measuring the change in particle size after perturbing the system by FD-RH. Figure 6 illustrates the ratios of particle size for $\mathrm{CH}$-coated or uncoated liposomes to original values after FD-RH. The higher values of particle size ratio for uncoated liposomes suggest aggregation and/or fusion of liposomal particles during FD-RH. When liposomes are cooled to a temperature where ice crystals form in the aqueous phase, the liposome particles are forced closer together, leading to the fusion of the particles. ${ }^{25}$ The addition of $\mathrm{CH}$ improved the stability of liposomes against severe physical stress $\left(-70^{\circ} \mathrm{C}\right)$. As the CH's concentration increased from $0 \%$ to $0.3 \%$, the values of particle size ratio decreased from 20 to 3.5. Chitosan contains hydroxyl groups along its backbone which can form hydrogen bonds between the polymer and water molecules. A similar protective mechanism for the aggregation and/or fusion of liposomes during freezing or dehydration as in the case of carbohydrates is expected for the polymer layer on the surface of liposomes. ${ }^{23}$ In addition, the protective effect of $\mathrm{CH}$ was partially attributed to the increased steric repulsion between the particles. ${ }^{28}$ Furthermore, after dehydration, $\mathrm{CH}$ formed the "wall material" surrounding the liposomal particles in the powder by forming a puseudohydration phase through their interaction

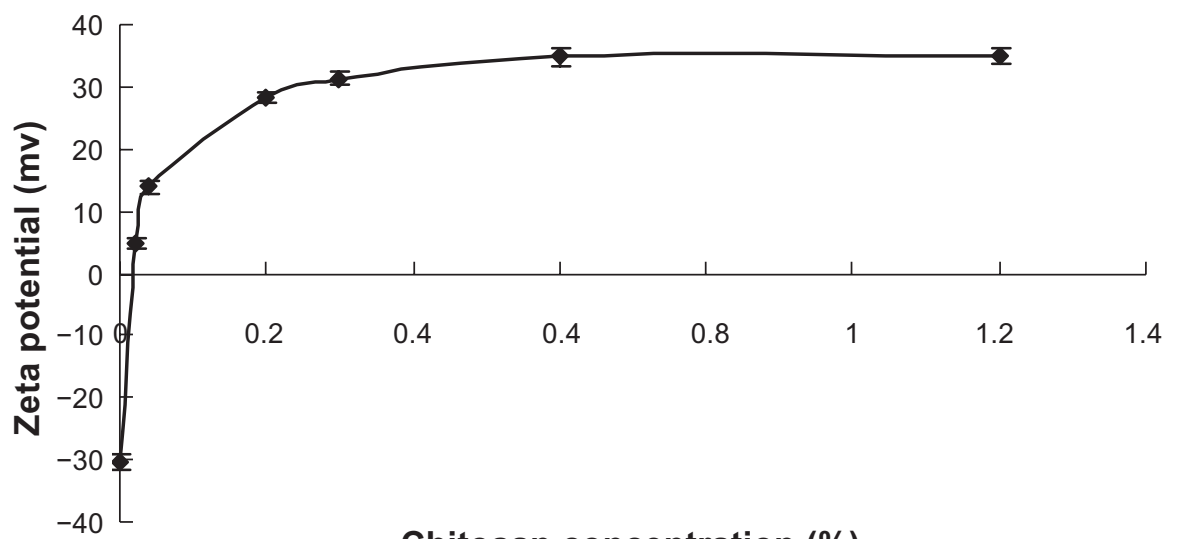

Chitosan concentration (\%)

Figure 3 The influence of $\mathrm{CH}$ concentration on the zeta potential of $\mathrm{CH}$-coated liposomes $(\mathrm{n}=3)$. 


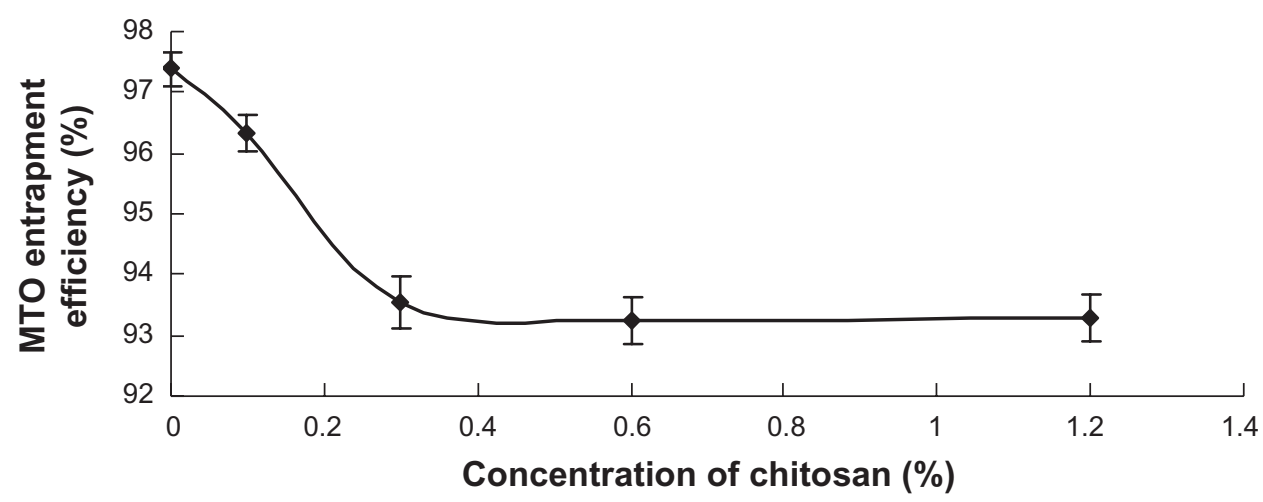

Figure 4 The effect of $\mathrm{CH}$ concentration on the entrapment efficiency of MTO $(n=3)$.

with phospholipid head groups, ${ }^{25}$ further improving the stability of the particles.

As the concentration of $\mathrm{CH}$ further increased from $0.3 \%$ to $1.2 \%$, the protective effect against the physical stress with FD-RH slightly decreased. The values of particle size ratio increased from $3.5(0.3 \% \mathrm{CH})$ to $6.25(1.2 \% \mathrm{CH})$. Since the liposomal membrane was fully covered with $\mathrm{CH}$ molecules, the adsorbed polymer is sufficiently strong to prevent particle-particle interaction by steric stabilization, and the excess $\mathrm{CH}$ would not help to further increase the stability of liposomes. On the contrary, the stability of liposomes would be slightly impaired. Laye et $\mathrm{al}^{29}$ have proposed the so-called saturation concentration $\left(\mathrm{C}_{\text {sat }}\right)$, which is the minimum concentration of polymer required to cover the oppositely charged particles, and an empirical model to calculate it via the change in zeta potential: ${ }^{25}$

$$
\zeta(c)=\zeta_{s a t}+\left(\zeta_{0}-\zeta_{s a t}\right) \exp \left(\frac{-3 c}{c_{s a t}}\right)
$$

where $\zeta(\mathrm{c})$ is the zeta potential of liposomes at a $\mathrm{CH}$ concentration of $\mathrm{c}$, and $\zeta_{0}$ and $\zeta_{\text {sat }}$ are the initial and saturation liposomal charges, respectively. According to the equation, the calculated $\mathrm{C}_{\text {sat }}$ was $0.28 \%$, which well agreed with the result we obtained in the zeta potential measurement $(0.3 \%)$. According to Laye's explanation, addition of $\mathrm{CH}$ to liposomes below and above the saturation concentration can both lead to the destabilization of liposomal dispersions. ${ }^{29} \mathrm{At}$ insufficient polymer concentrations, the anionic phospholipid molecules of liposomes may have been bound to the cationic $\mathrm{CH}$ molecules to form coacervates rather than the cationic $\mathrm{CH}$ molecules wrapping themselves around the surfaces of the liposome particles. At excess polymer concentrations, the exclusion of polymer molecules from a narrow region surrounding the particle surfaces generates an attractive force that is strong enough to overcome the inter-molecular repulsive forces and to bring the particles together, making the liposomes susceptible to depletion flocculation. ${ }^{25,29}$ The narrow region (ie, "depletion zone") was formed due to the conformational entropy restrictions. The "zone" existed

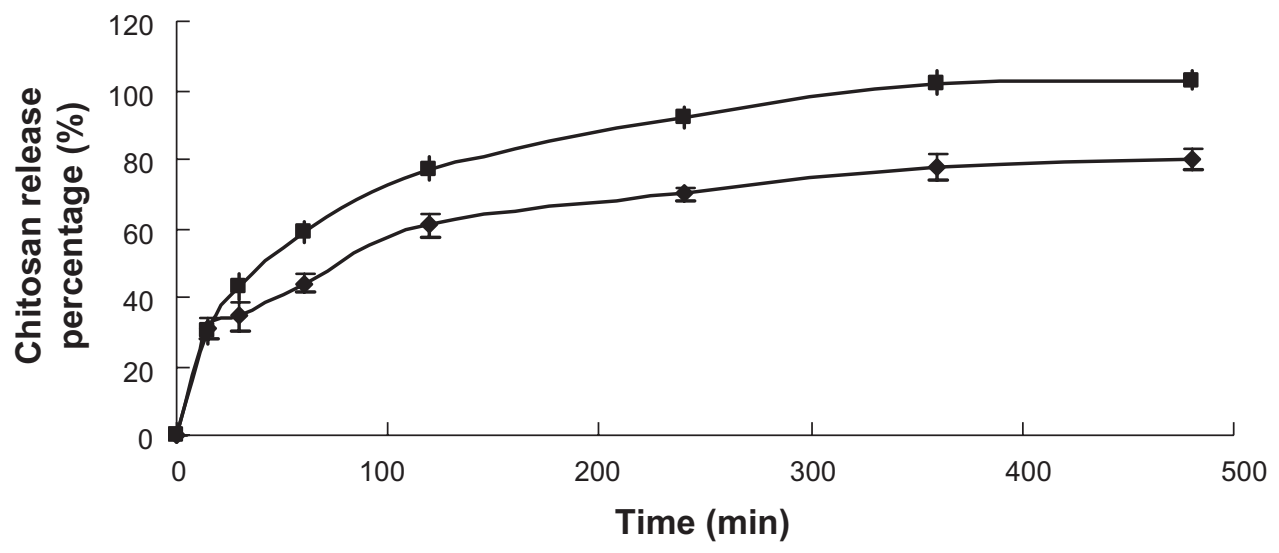

Figure 5 The desorption of $\mathrm{CH}$ from the coated liposomes $(\bullet)$ using $\mathrm{CH}$ solution as control $(\mathbf{m})(\mathrm{n}=3)$. 


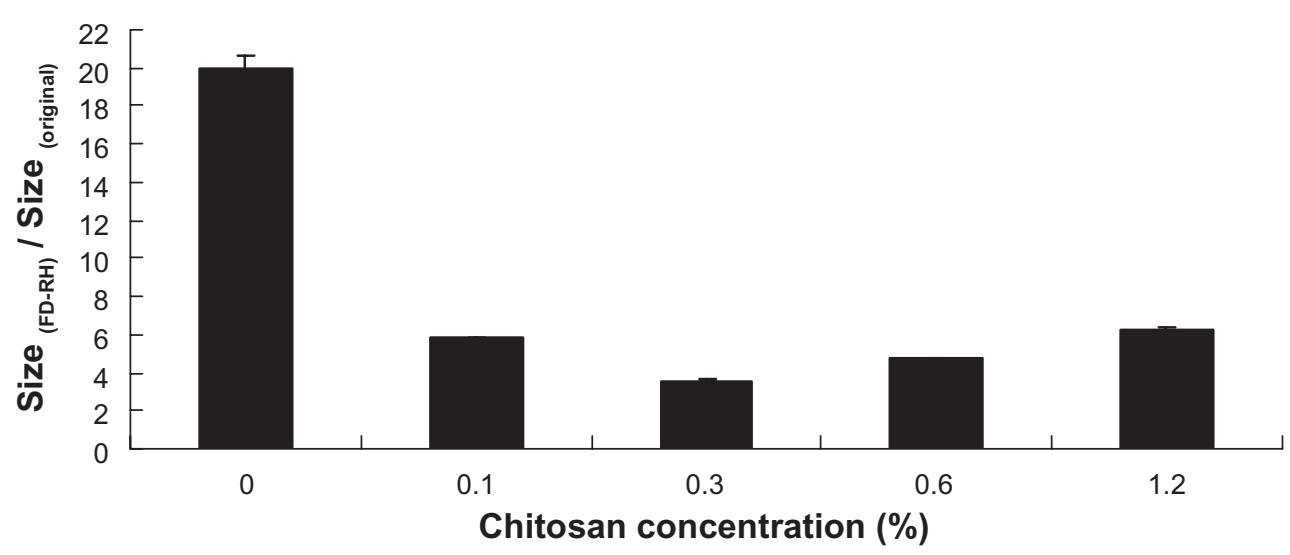

Figure 6 Relative particle size of liposomes coated with $\mathrm{CH}$ after FD-RH compared to that before FD-RH.

between the surface of chitosan-coated particles and the surface in contact with a solution of non-adsorbing polymer. The polymer concentration in the depletion zone is lower than that of the bulk solution, resulting in an attractive force between the particles, which is proportional to the osmotic pressure of the polymer in the bulk solution. . $^{30,31}$

\section{In vitro drug release}

In the in vitro release study, an aqueous solution of MTO was released rapidly and almost completed within $7 \mathrm{~h}$. However, the uncoated and $\mathrm{CH}$-coated liposomes $(\mathrm{CH}$ concentration $0.3 \%$ and $0.6 \%$ ) released $31.39 \%-54.10 \%$ of MTO within $48 \mathrm{~h}$ of dialysis as shown in Figure 7. The encapsulation of MTO into liposomes significantly slowed its release indicating the depot effect of liposomes, especially the CH-coated liposomal formulations. The sustained release behavior of MTO from polymer-coated liposomes was attributed to the retarded effect of $\mathrm{CH}$ coating and the stabilization of coated liposomes in the serum. Besides, $\mathrm{CH}$ at a concentration of $0.3 \%$ had a better protective effect over $\mathrm{CH}$ at a concentration of $0.6 \%$, which was in excellent agreement with the result obtained in FD-RH stability studies ("Physical stability of CH-coated liposomes").

\section{Pharmacokinetics of the $\mathrm{CH}$-coated liposomes after intravenous injection in rats}

Figure 8 depicts the plasma level-time profiles of MTO saline solution, uncoated liposomes and $\mathrm{CH}$-coated liposomes after intravenous injection in rats. $\mathrm{CH}$ at a concentration of $0.3 \%$ and $0.6 \%$ were used to study the polymer coating effect on the systemic circulation of the liposomes. A rapid decrease of the drug from the systemic circulation for MTO solution was observed within 15 minutes, followed by a relatively slower and steady decrease. After 2 hours, the plasma concentration decreased to around the detection limit $(20 \mathrm{ng} / \mathrm{mL})$. Encapsulation of MTO in the liposomes significantly increased the retention time and plasma concentration $(P<0.05)$. The area under the curve (AUC) for the uncoated liposomes was 7.35-fold of that for the MTO solution (calculated from

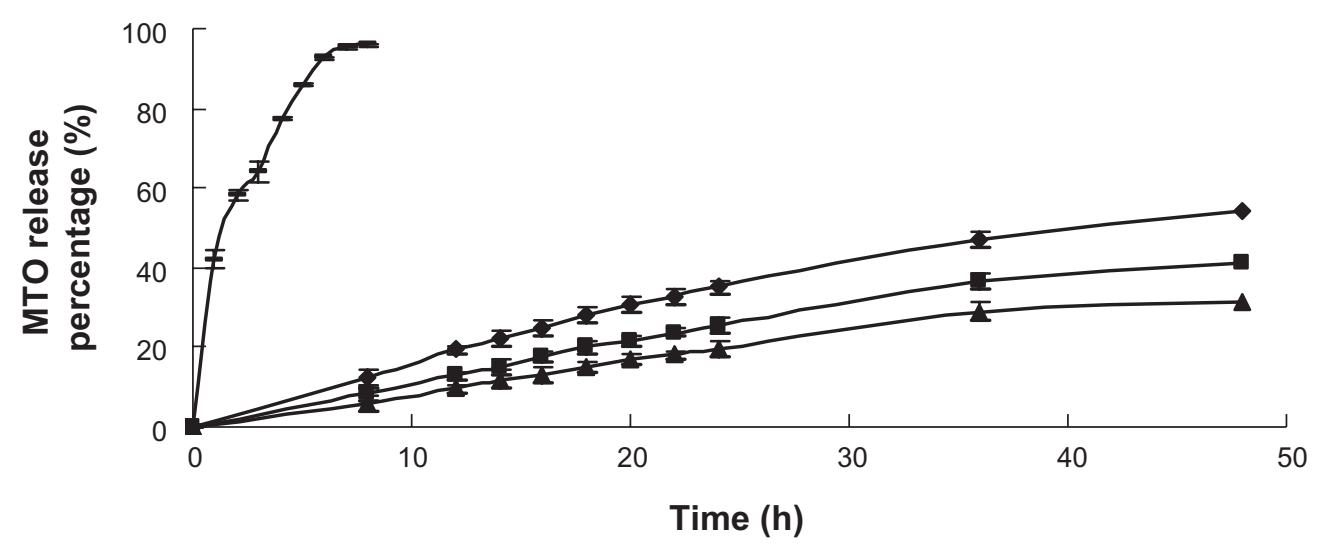

Figure 7 In vitro release of MTO from uncoated liposomes $(\bullet), \mathrm{CH}$-coated liposomes $(0.6 \%)(\boldsymbol{\bullet})$, and $\mathrm{CH}$-coated liposomes $(0.3 \%)(\mathbf{\Delta})$ in serum using MTO aqueous solution as control $(-)(n=3)$. 


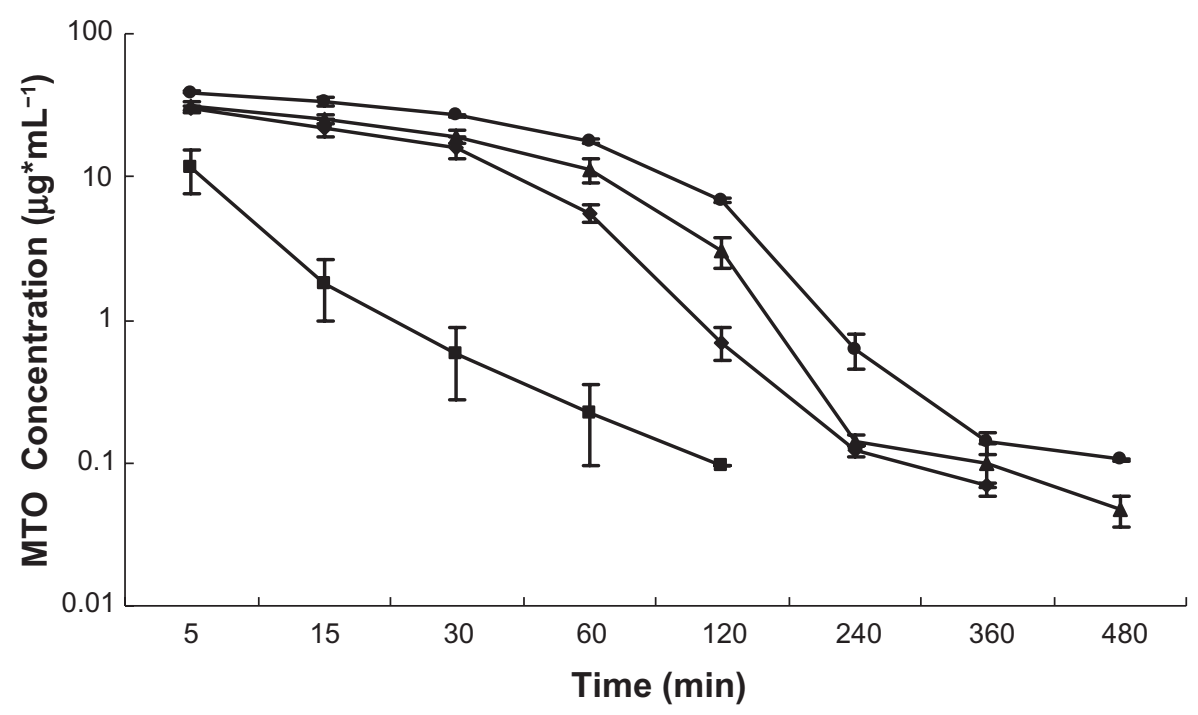

Figure 8 Semi-log plasma concentration-time profiles in rats after intravenous administration of MTO solution ( $\mathbf{\square})$, uncoated liposomes $(\bullet)$, CH-coated liposomes $(0.6 \%)$ $(\mathbf{A})$, and $\mathrm{CH}$-coated liposomes $(0.3 \%)(\bullet)(\mathrm{n}=6)$.

the data shown in Table 1). Comparing the pharmacokinetic profiles of the uncoated liposomes and $\mathrm{CH}$-coated liposomes, an even higher retention time and plasma concentration were observed for the latter, confirming the effectiveness of the steric coating layer of $\mathrm{CH}$ formed on the liposomal surface for prolonging the retention time of the carriers.

However, as the concentration of $\mathrm{CH}$ increased from $0.3 \%$ to $0.6 \%$, the retention of the liposome did not increase accordingly, on the contrary, a decrease of both AUC and MRT were observed. The AUC and MRT value for $\mathrm{CH}$-coated liposomes at a concentration of $0.3 \%$ was 1.60 -fold and 1.30 -fold of that for $\mathrm{CH}$ at a concentration of $0.6 \%$, respectively (calculated from the data shown in Table 1). This is in agreement with the result in physical stability and in vitro release studies, the excess $\mathrm{CH}$ leading to the decreased protective effect for liposomes, which means the dominant mechanism of prolonged retention of MTO in CH-coated liposomes is to stabilize the lipid bilayer membranes after entering the systemic circulation.

\section{Conclusion}

$\mathrm{CH}$ formed a hydrophilic layer on the surface of liposomes by a simple method of mixing a liposomal suspension with a polymer solution followed by incubation, resulting in particle size increment and positive zeta potential. The effectiveness of $\mathrm{CH}$ coating was confirmed by a sustained release of adsorbed $\mathrm{CH}$ from the liposomes compared with the $\mathrm{CH}$ solution. The enhancing physical stability of the resultant $\mathrm{CH}$-coated liposomes was observed by FD-RH. The presence of free excess $\mathrm{CH}$ would decrease the stability of the liposomes. By comparing the circulating profiles of various liposomes with different coating amounts of $\mathrm{CH}$, it was concluded that both the steric property of the coating layer and stabilization of the lipid bilayer membranes can affect the systemic circulation of the liposomes.

Polymer coating is an easy and flexible way to alter the surface properties of the liposomes, and $\mathrm{CH}$, one of the most promising coating materials, is expected to be fully examined.

\section{Acknowledgments/disclosures}

The authors report no conflicts of interest in this work. This study is financially supported by National Natural Science Foundation of China (Project No. 30873183).

Table I Mean pharmacokinetic parameters of MTO after intravenous administration of MTO solution, uncoated liposomes, $\mathrm{CH}(0.3 \%)$ and $\mathrm{CH}(0.6 \%)$-coated liposomes

\begin{tabular}{lccc}
\hline Parameters & Solution & Uncoated liposomes & $\begin{array}{l}\text { CH-coated liposomes } \\
\mathbf{( 0 . 6 \% )}\end{array}$ \\
\hline AUC $\left(\mu g^{*} \mathrm{~mL}^{-1 *} \min \right)$ & $162.33 \pm 12.10$ & $1193.50 \pm 143.27^{*}$ & $1709.09 \pm 203.53^{*, \#}$ \\
MRT $(\min )$ & $18.73 \pm 0.52$ & $35.67 \pm 4.76^{*}$ & $47.74 \pm 0.96^{*, \#}$ \\
$\mathrm{t}_{1 / 2}(\min )$ & $19.33 \pm 1.23$ & $35.07 \pm 5.25^{*}$ & $46.73 \pm 2.84^{*, \#}$ \\
\hline
\end{tabular}

Notes: Each value is the mean \pm standard deviation, $n=6$. ${ }^{*} P<0.05$, compared with MTO solution; ${ }^{*} P<0.05$, compared with uncoated liposomes; ${ }^{*} P<0.05$, compared with $\mathrm{CH}$-coated liposomes (0.6\%). 


\section{References}

1. Chang CC, Liu DZ, Lin SY, et al. Liposome encapsulation reduces cantharidin toxicity. Food Chem Tox. 2008;46(9):3116-3121.

2. Martins S, Sarmento B, Ferreira DC, Souto EB. Lipid-based colloidal carriers for peptide and protein delivery - liposomes versus lipid nanoparticles. Int J Nanomed. 2007;2(4):595-607.

3. Pajean M, Huc A, Herbage D. Stabilization of liposomes with collagen. Int J Pharm. 1991;77(1):31-40.

4. Senior JH. Fate and behavior of liposomes in vivo: a review of controlling factors. Crit Rev Ther Drug Carrier Syst. 1987;3(2):123-193.

5. Storm G, Belliot SO, Daemeh T, Lasic DD. Surface modification of nanoparticles to oppose uptake by the mononuclear phagocyte system. Adv Drug Deliv Rev. 1995;17(1):31-48.

6. Torchilin VP, Trubetskoy VS. Which polymers can make nanoparticulate drug carriers long-circulating. Adv Drug Deliv Rev. 1995;16(2-3):141-155.

7. Chonn A, Cullis PR, Devine DV. The role of surface charge in the activation of the classical and alternative pathways of complement by liposomes. J Immunol. 1991;146(12):4234-4241.

8. Juliano RL, Stamp D. The effect of particle size and charge on the clearance rates of liposomes and liposome encapsulated drugs. Biochem Biophys Res Commun. 1975;63(3):651-658.

9. Patel HM. Serum opsonins and liposomes: their interaction and opsonophagocytosis. Crit Rev Ther Drug Carrier Syst. 1992;9(1): 39-90.

10. Chonn A, Semple SC, Cullis PR. Association of blood proteins with large unilamellar liposomes in vivo. Relation to circulation lifetimes. J Biol Chem. 1992;267(26):18759-18765.

11. Takeuchi H, Kojima H, Yamamoto H, Kawashima Y. Polymer coating of liposomes with a modified polyvinyl alcohol and their systemic circulation and RES uptake in rats. J Control Rel. 2000;68(2):195-205.

12. $\mathrm{Mu} \mathrm{X}$, Zhong $\mathrm{Z}$. Preparation and properties of poly(vinyl alcohol)stabilized liposomes. Int J Pharm. 2006;318(1-2):55-61.

13. Cheng J, Zhu JB, Wen N, Xiong F. Stability and pharmacokinetic studies of $O$-palmitoyl amylopectin anchored dipyridamole liposomes. Int J Pharm. 2006;313(1-2):136-143.

14. Ravi Kumar MNV. A review of chitin and chitosan applications. React Funct Polym. 2000;46(1):1-27.

15. Borchard G, Junginger HE. Modern drug delivery applications of chitosan. Adv Drug Deliv Rev. 2001;52(2):103.

16. Ugwu S, Zhang A, Parmar M, Miller B, Sardone T, Peikov V, Ahmad I. Preparation, characterization, and stability of liposome-based formulations of mitoxantrone. Drug Dev Ind Pharm. 2005;31(2):223-229.

17. Duan Y, Yu B, Zhang Z. Preparation of long circulating mitoxantrone liposomes and its pharmacokinetics. Acta Pharm Sinica. 2002;37(6):465-468.
18. Rengel RG, Barisic K, Pavelic Z, Grubisic TZ, Cepelak I, Filipovic-Grcic J. High efficiency entrapment of superoxide dismutase into mucoadhesive chitosan-coated liposomes. Eur J Pharm Sci. 2002;15(5):441-448.

19. Gao GZ, Jiao QC, Ding YL, Chen L. Study on quantitative assay of chitosan in compound sample with a spectrophotometric method. Food and Ferm Ind. 2003;29(5):49-52.

20. Johnson JL, Ahmad A, Khan S, et al. Improved liquid chromatographic method for mitoxantrone quantification in mouse plasma and tissues to study the pharmacokinetics of a liposome entrapped mitoxantrone formulation. J Chromatogr B. 2004;799(1):149-155.

21. Blakmeer J, Behmer MR, Cohen Stuart MA, Fleer GJ. Adsorption of weak polyelectrolytes on highly charged surfaces: polyfacrylic acid on olystyrene latex with strong cationic groups. Macromol. 1990;23(8):2301-2309.

22. Henriksen I, Smistad G, Karlsen. Interactions between liposomes and chitosan. Int J Pharm. 1994;101(3):227-236.

23. Takeuchi H, Yamamoto H, Toyoda T, Toyobuku H, Hino T, Kawashima Y. Physical stability of size controlled small unilameller liposomes coated with a modified polyvinyl alcohol. Int J Pharm. 1998;164(1-2):103-111.

24. Schonhoff M. Layered polyelectrolyte complexes: physics of formation and molecular properties. J Phys Condens Matter. 2003;15(49):1781-1808.

25. Guzey D, McClements DJ. Formation, stability and properties of multilayer emulsions for application in the food industry. Adv Colloid Interface Sci. 2006;128-130(21):227-248.

26. Guo J, Ping Q, Jiang G, Huang L, Tong Y. Chitosan-coated liposomes: characterization and interaction with leuprolide. Int J Pharm. 2003;260(2):167-173.

27. Fang N, Chan V, Mao HQ, Leong KW. Interactions of phospholipid bilayer with chitosan: effect of molecular weight and pH. Biomacromol. 2001;2(4):1161-1168.

28. Kellaway IW, Najib NM. Hydrophilic polymers as stabilizers and flocculants of sulphadimidine suspensions. Int J Pharm. 1981;9(1):59-66.

29. Laye C, McClements DJ, Weiss J. Formation of biopolymer-coated liposomes by electrostatic deposition of chitosan. J Food Sci. 2008; 73(5):N7-N15.

30. Jenkins P, Snowden M. Depletion flocculation in colloidal dispersions. Adv Colloid Interface Sci. 1996;68(1):57-96.

31. Kwaambwa HM. Stability and phase separation behavior of systems of particles in a medium with added polymer. Water SA. 2006;32(1):111-114
International Journal of Nanomedicine

\section{Publish your work in this journal}

The International Journal of Nanomedicine is an international, peerreviewed journal focusing on the application of nanotechnology in diagnostics, therapeutics, and drug delivery systems throughout the biomedical field. This journal is indexed on PubMed Central, MedLine, CAS, SciSearch $\AA$, Current Contents ${ }^{\circledR} /$ Clinical Medicine,

\section{Dovepress}

Journal Citation Reports/Science Edition, EMBase, Scopus and the Elsevier Bibliographic databases. The manuscript management system is completely online and includes a very quick and fair peer-review system, which is all easy to use. Visit http://www.dovepress.com/ testimonials.php to read real quotes from published authors. 